\title{
Life-Threatening Constipation Induced by Intrathecal Baclofen Therapy
}

\author{
Francesco Patti Angelo Pappalardo Salvatore Lo Fermo Vincenzo Cimino \\ Alberto Castiglione Mario Zappia \\ Department of Neurosciences, University of Catania, Catania, Italy
}

Dear Sir,

Intrathecal baclofen (ITB) delivery is a therapeutic option for patients with severe spasticity not responding to common oral medications, such as baclofen, dantrolene or tizanidine. At common doses, ITB is usually tolerated by patients [1]. Unfortunately, higher doses could be necessary to treat many cases of spasticity, thus enhancing the frequency of side effects [2-4]. Concerning gastrointestinal function, ITB could affect peristalsis, which could be severely slowed down to paralytic ileus. Nevertheless, constipation has previously been reported as an infrequent ITB-induced adverse effect, ranging from 3 to $10 \%$ of treated patients [2-4]. Here we report that, in our series of patients treated with ITB, constipation was a frequent and serious side effect, leading to death in 1 case.

Thirty-eight patients (26 men and 12 women; age $48.7 \pm 15.4$ years, mean \pm SD) were implanted for continuous ITB infusion from August 2002 to July 2006. They had severe spasticity due to different neurological diseases. Thirty-six patients out of 38 (95\%) were affected by spinal cord diseases (myelitis, $\mathrm{n}=19$; multiple sclerosis, $\mathrm{n}=13$; traumatic injury, $\mathrm{n}=4$ ). The remaining 2 patients suffered from spasticity due to anoxic encephalopathy. All patients had previously been treated with oral baclofen, dantrolene, or tizanidine at their optimal doses, without relevant clinical benefit. Twenty-three out of 38 patients $(61 \%)$ were on treatment with other drugs (4 azathioprine, 2 methotrexate, 1 interferon beta- 1 b, 8 gabapentin, 2 pregabalin, 2 phenobarbital, 2 paroxetine, 1 citalopram, 1 venlafaxine, 7 furosemide, 4 heparin, 1 risperidone and 1 olanzapine). Fifteen patients did not receive any concomitant medication.

ITB was administered with a mean effective daily dose of $251.3 \pm 11 \mu \mathrm{g}$ (range 90-580 $\mu \mathrm{g}$ ). Adverse events of ITB were monitored by evaluating patients' monthly records and constipation was defined as a reduced frequency of evacuation (2 or fewer acts in a week) [5].

Within 6 months after ITB implantation, all patients had an optimal therapeutic response to ITB and mean Ashworth score decreased from $3.4 \pm 1.1$ to $1.6 \pm 1.1$ points $(\mathrm{p}<0.0001)$. Figure 1 shows the frequency of side effects. Constipation was the most frequent side effect, being reported by all patients, even if 9 subjects (24\%) already suffered from constipation before implantation. Nevertheless, ITB further

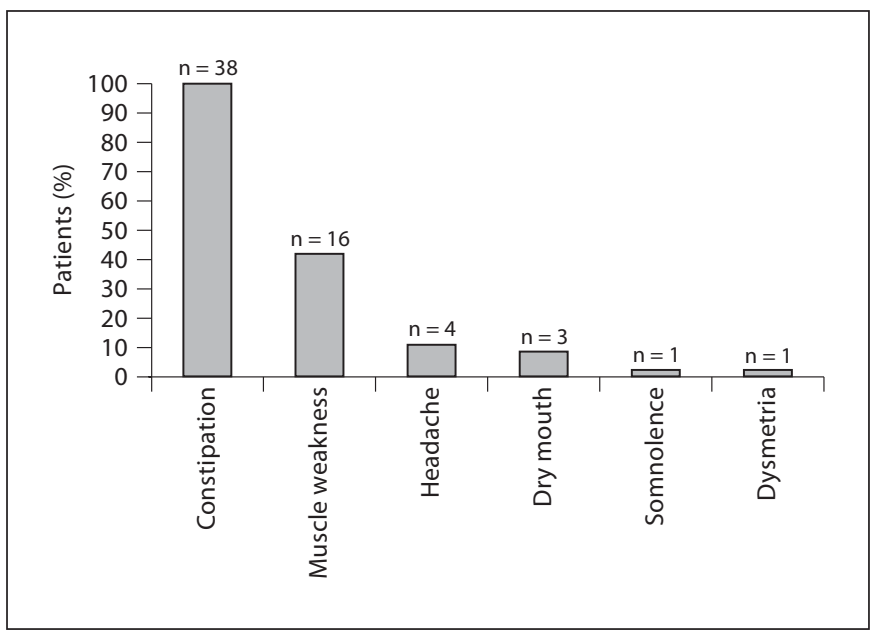

Fig. 1. Adverse events reported by patients after beginning ITB therapy. 
worsened constipation, reducing bowel evacuation from $2.5 \pm 0.7$ to $0.6 \pm 0.6$ acts per week $(\mathrm{p}<0.01)$. During the followup period (32.1 \pm 8.7 months, range 6$40)$, paralytic ileum with abdominal distension occurred in 3 patients. One of them died from complications partly due to the surgical management, carried out for obstructive ileus. The deceased patient was a 62 -year-old woman affected by myelitis and treated with ITB at a daily dose of $132 \mu \mathrm{g}$, who developed vomiting, epigastralgia and absence of peristalsis 7 months after the implantation. Her last bowel evacuation had occurred 24 days before. She had an abdominal X-ray examination which revealed ileal occlusion due to accumulation of feces. It could be hypothesized that peristalsis was mainly slowed down by ITB. This effect could be responsible for the accumulation of feces and then obstructive ileus.

Impairment of bowel evacuation could be due to the effects of baclofen on the enteric nervous system. The activation of $\mathrm{GABA}_{\mathrm{B}}$ receptors by their agonist (baclofen) can lead to the inhibition of electrically evoked contractions of the myen- teric plexus-longitudinal muscle [6]. Baclofen could also exert an agonist effect on $\mathrm{GABA}_{\mathrm{B}}$ receptors of the nucleus tractus solitarii and may have an inhibitory effect in vagal nuclei, leading to a suppression of vagal reflexes [7].

Our data do not support previous studies, in which the frequency of constipation was low, accounting for up to $10 \%$ of patients, or not reported [1-4]. One possible explanation of the discrepancies between our results and those of others [1-4] could be a bias of ascertainment in patients who are already constipated before implantation or not examined for this condition. Nevertheless, recognition of constipation in patients treated with ITB is relevant, because constipation is not only a frequent side effect, being present in every patient of our series, but it is also a life-threatening complication, considering that about $8 \%$ of our patients had paralytic ileum, responsible for the death of 1 woman. We recommend that the management of patients treated with ITB should take into account not only eventual benefits but also possible frequent and life-threatening side effects.

\section{References}

1 Zahavi A, Geertzen JHB, Middel B, Staal M, Rietman JS: Long-term effect (more than five years) of intrathecal baclofen on impairment, disability and quality of life in patients with severe spasticity of spinal origin. J Neurol Neurosurg Psychiatry 2004;75: 1553-1557.

-2 Emery E: Intrathecal baclofen: literature review of the results and complications. Neurochirurgie 2003;49:276-288.

3 Kofler M, Matzak H, Saltuari L: The impact of intrathecal baclofen on gastrointestinal function. Brain Injury 2002;16:825-836.

$\checkmark 4$ Ben Smail D, Peskine A, Roche N, Mailhan L, Thiebaut JB, Bussel B: Intrathecal baclofen for treatment of spasticity of multiple sclerosis patients. Mult Scler 2006;12:101-103.

5 Corazziari E: Definition and epidemiology of functional gastrointestinal disorders. Best Pract Res Clin Gastroenterol 2004;18:613631.

6 6 Begg M, Molleman A, Parsons M: Modulation of the release of endogenous $\gamma$-aminobutyric acid by cannabinoids in the guinea pig ileum. Eur J Pharmacol 2002;434:87-94.

7 Davis SF, Derbenev AV, Williams KW, Glatzer NR, Smith BN: Excitatory and inhibitory local circuit input to the rat dorsal motor nucleus of the vagus originating from the nucleus tractus solitarius. Brain Res 2004; 1017:208-217. 